\title{
Transmural heterogeneity of myofilament function and sarcomeric protein phosphorylation in remodeled myocardium of pigs with a recent myocardial infarction
}

\section{Jolanda van der Velden ${ }^{1}$ *, Daphne Merkus ${ }^{2}$, Vincent de Beer ${ }^{2}$, Nazha Hamdani ${ }^{1,3}$, Wolfgang A. Linke ${ }^{3}$, Nicky M. Boontje ${ }^{1}$, Ger J. M. Stienen ${ }^{1,4}$ and Dirk J. Duncker ${ }^{2}$}

1 Laboratory for Physiology, Institute for Cardiovascular Research, VU University Medical Center, Amsterdam, Netherlands

2 Experimental Cardiology, Thoraxcenter, Cardiovascular Research school COEUR, Erasmus MC, University Medical Center, Rotterdam, Netherlands

${ }^{3}$ Department of Cardiovascular Physiology, Institute of Physiology, Ruhr University Bochum, Bochum, Germany

${ }^{4}$ Department of Physics and Astronomy, VU University, Amsterdam, Netherlands

Edited by:

Kenneth S. Campbell, University of Kentucky, USA

\section{Reviewed by:}

Martina Krüger, Ruhr University

Bochum, Germany

Andrew Trafford, University of

Manchester, UK

${ }^{*}$ Correspondence:

Jolanda van der Velden, Laboratory for Physiology, VU University Medical Center, van der Boechorststraat 7, 1081 BT, Amsterdam, Netherlands. e-mail: j.vandervelden@vumc.nl
Aim: Transmural differences in sarcomeric protein composition and function across the left ventricular (LV) wall have been reported. We studied in pigs sarcomeric function and protein phosphorylation in subepicardial (EPI) and subendocardial (ENDO) layers of remote LV myocardium after myocardial infarction (MI), induced by left circumflex coronary artery ligation. Methods: EPI and ENDO samples were taken 3 weeks after sham surgery $(n=12)$ or induction of $\mathrm{MI}(n=12)$ at baseline (BL) and during $\beta$-adrenergic receptor ( $\beta \mathrm{AR})$ stimulation with dobutamine. Isometric force was measured in single cardiomyocytes at various $\left[\mathrm{Ca}^{2+}\right]$ and $2.2 \mu \mathrm{m}$ sarcomere length. Results: In sham hearts, no significant transmural differences were observed in myofilament function or protein phosphorylation. Myofilament $\mathrm{Ca}^{2+}$-sensitivity was significantly higher in both EPI and ENDO of MI compared to sham hearts. Maximal force was significantly reduced in $\mathrm{Ml}$ compared to sham, but solely in ENDO cells. A higher passive force was observed in MI hearts, but only in EPI cells. The proportion of stiff N2B isoform was higher in EPI than in ENDO in both sham and MI hearts, and a trend toward increased N2B-proportion appeared in MI EPI, but not MI Endo. Analysis of myofilament protein phosphorylation did not reveal significant transmural differences in phosphorylation of myosin binding protein $\mathrm{C}$, desmin, troponin T, troponin I (cTnl), and myosin light chain 2 (MLC-2) both at BL and during $\beta A R$ stimulation with dobutamine infusion. A significant increase in MLC-2 phosphorylation was observed during dobutamine only in sham. In addition, the increase in cTnl phosphorylation upon dobutamine was twofold lower in MI than in sham. Conclusion: Myofilament dysfunction is present in both EPI and ENDO in post-MI remodeled myocardium, but shows a high degree of qualitative heterogeneity across the LV wall. These heterogeneous transmural changes in sarcomeric properties likely contribute differently to systolic vs. diastolic global LV dysfunction after MI.

Keywords: sarcomere function, myocardial infarction, heart, subendocardium, subepicardium, protein phosphorylation

\section{INTRODUCTION}

Upon electrical activation of cardiac muscle, cells calcium is released from the sarcoplasmic reticulum which initiates contraction of the sarcomeres upon binding of calcium to the troponin complex. This process of excitation-contraction coupling involves tight regulation of mechanisms involved in excitation, calcium handling, and sarcomeric contraction. Excitation of the healthy heart is characterized by heterogeneity across the ventricular wall evident from the difference in duration of the action potential between subepicardial and subendocardial ventricular cells (Litovsky and Antzelevitch, 1989; Glukhov et al., 2010). This heterogeneity underlies optimal timing of ventricular excitation, and loss of this electrophysiologic heterogeneity is thought to contribute to arrhythmias in cardiac disease (Glukhov et al., 2010).
Apart from heterogeneity in excitation, transmural differences have been reported in mechanisms involved in sarcomeric contraction. For example, a transmural gradient in myosin light chain 2 (MLC-2) phosphorylation has been reported in rodent studies (Davis et al., 2001; Cazorla et al., 2005; Rajashree et al., 2005), which has been proposed to underlie the wringing motion (i.e., torsion) of the heart to eject blood (Davis et al., 2001). A study in pig myocardium showed subtle differences in the composition of myosin heavy chain isoforms across the ventricular wall (Stelzer et al., 2008). A significantly higher expression of the fast $\alpha$-myosin heavy chain isoform in subepicardial fibers (13\% of total MHC) compared to subendocardial fibers ( $3 \%$ of total MHC) correlated with faster rates of delayed force development and force decay, and may underlie appropriate timing of force generation across the ventricular wall (Stelzer et al., 2008). 
To our knowledge there are no studies available that investigated whether transmural differences exist in the responses of myofilament function and sarcomeric protein phosphorylation to cardiac pathology in large mammals. In previous studies (Van der Velden et al., 2004; Duncker et al., 2009; Boontje et al., 2011) we found a reduced force-generating capacity and an increased $\mathrm{Ca}^{2+}$-sensitivity in cardiomyocytes isolated from postinfarct subendocardial remodeled myocardium. In the present study we investigated if alterations in sarcomeric functional properties are different between the subepi- and subendo-cardial layer of remodeled myocardium 3 weeks after myocardial infarction in a pig model. As phosphorylation of sarcomeric proteins is an important regulator of myocardial contraction, and perturbations in phosphorylation frequently underlie impaired cardiac function in cardiac pathologies, force measurements in single Tritonpermeabilized cardiomyocytes were combined with analysis of the transmural phosphorylation pattern of sarcomeres. Moreover, to investigate transmural protein phosphorylation patterns during cardiac stress, as occurs upon activation of the $\beta$-adrenergic receptor pathway, biopsies were obtained during dobutamine stimulation.

\section{MATERIALS AND METHODS MYOCARDIAL INFARCTION MODEL}

Experiments were performed in accordance with the Guide for the Care and Use of Laboratory Animals (NIH Publication 86-23, revised 1996), and with approval of the local ethics committee for animal experiments approved procedures. Twenty-four 23 month old Yorkshire-Landrace pigs of either sex entered the study. Pigs were sedated (ketamine, $20 \mathrm{mg} / \mathrm{kg} \mathrm{IM}$, and midazolam, $0.5 \mathrm{mg} / \mathrm{kg}$ IM), anesthetized (thiopental, $10 \mathrm{mg} / \mathrm{kg} \mathrm{IV}$ ), intubated, and ventilated with $\mathrm{O}_{2}$ and $\mathrm{N}_{2} \mathrm{O}$ to which $0.1-1 \%$ (vol/vol) isoflurane was added (Van Kats et al., 2000; Haitsma et al., 2001). Anesthesia was maintained with midazolam $(2 \mathrm{mg} / \mathrm{kg}$ followed by $1 \mathrm{mg} / \mathrm{kg} / \mathrm{h} \mathrm{IV})$ and fentanyl $(10 \mu \mathrm{g} / \mathrm{kg} / \mathrm{h} \mathrm{IV})$. Under sterile conditions, the chest was opened via the fourth left intercostal space and a $4 \mathrm{~cm}$ incision was made in the pericardium at the site of the origin of the left circumflex coronary artery (LCx). Then, the LCx was dissected out and a suture was placed around it. The LCx was permanently ligated to produce a myocardial infarction (MI, $n=12)$, whereas in the animals from the sham group $(n=12)$ the suture was removed. The chest was closed and the animals were allowed to recover, receiving analgesia ( $0.3 \mathrm{mg}$ buprenorphine IM) for 2 days and antibiotic prophylaxis $(25 \mathrm{mg} / \mathrm{kg}$ amoxicillin and $5 \mathrm{mg} / \mathrm{kg}$ gentamycin IV) for 5 days.

\section{IN VIVO MEASUREMENTS AND STORAGE OF CARDIAC TISSUE SAMPLES}

Three weeks after surgery, pigs were sedated (ketamine, $20 \mathrm{mg} / \mathrm{kg}$ IM and midazolam, $0.5 \mathrm{mg} / \mathrm{kg} \mathrm{IM}) .2 \mathrm{D}$ echocardiographic recordings of the left ventricular (LV) short axis at midpapillary level were obtained (ALOKA ProSound SSD-4000; Japan) and stored for offline analysis (Van Kats et al., 2000; Van der Velden et al., 2004). LV end-diastolic cross-sectional area and 2-D LV end-systolic cross-sectional area were determined, and LV ejection fraction was calculated as (end-diastolic area - end-systolic area)/end-diastolic area $\times 100 \%$.
Subsequently, pigs were anesthetized (pentobarbital, $20 \mathrm{mg} / \mathrm{kg}$ IV), intubated and ventilated with $\mathrm{O}_{2}$ and $\mathrm{N}_{2}$ (Van Kats et al., 2000; Van der Velden et al., 2004). Anesthesia was maintained with pentobarbital (10-15 mg/kg/h IV). Animals were instrumented to allow closed-chest monitoring of heart rate, cardiac output, mean aortic and pulmonary artery blood pressures, LV pressure and its first derivative $\mathrm{dP} / \mathrm{dt}$, to assess $\mathrm{dP} / \mathrm{dt}_{\max }$ and indices of diastolic function, including $\mathrm{LVdP} / \mathrm{dt}_{\min }$, Tau and $\mathrm{LV}$ end-diastolic pressure (LVEDP; Van Kats et al., 2000; Van der Velden et al., 2004). Subsequently, a midline sternotomy was performed and the heart was suspended in a pericardial cradle. In six Sham and six MI swine, hearts were then arrested and immediately excised. Subepicardial (EPI) and subendocardial (ENDO) samples were obtained from remote non-infarcted myocardium of the LV anterior free wall and immediately frozen in liquid nitrogen.

To determine changes in myofilament protein phosphorylation upon $\beta$-adrenergic receptor stimulation, transmural needle (thrucut) biopsies were taken from 12 different animals (six sham and six MI) before (basal) and at the end of two consecutive $10 \mathrm{~min}$ intravenous (IV) infusions of dobutamine $(2$ and $10 \mu \mathrm{g} / \mathrm{kg} / \mathrm{min}$ : Dob2 and Dob10) from the LV anterior free wall myocardium (in MI pigs: remodeled non-infarcted tissue). The transmural biopsies were cut to obtain EPI and ENDO tissue samples, which were subsequently frozen and stored in liquid nitrogen. In MI pigs biopsies were taken randomly from anywhere in the remodeled anterior LV wall, covering as much as $30 \%$ of the non-infarcted left ventricle.

\section{CARDIOMYOCYTE MEASUREMENTS}

Single cardiomyocytes were obtained via mechanical isolation in cold relaxing solution containing (in $\mathrm{mM}$ ) free $\mathrm{Mg}^{2+} 1, \mathrm{KCl} 100$, EGTA 2, Mg-ATP 4, imidazole 10 (pH 7.0, adjusted with $\mathrm{KOH}$ ). Subsequently, cells were incubated for $5 \mathrm{~min}$ in relaxing solution with Triton X-100 (0.5\%) to remove all membranes as described previously (Boontje et al., 2011). Isometric force was measured at various calcium concentrations at $15^{\circ} \mathrm{C}$ and sarcomere length of $2.2 \mu \mathrm{m}$. The diameters of the cardiomyocyte were measured microscopically, in two perpendicular directions. Cross-sectional area was calculated assuming an elliptical cross-section. Relaxing and activating solutions for force measurements contained, respectively (in mM): $\mathrm{MgCl}_{2}: 6.48$ and 6.28, $\mathrm{Na}_{2} \mathrm{ATP}: 5.89$ and 5.97, EGTA: 7.0 and 0, CaEGTA: 0 and 7.0. In addition, both contained $14.5 \mathrm{mM}$ phosphocreatine and $60 \mathrm{mM}$ BES ( $\mathrm{pH} 7.1$, adjusted with $\mathrm{KOH}$; Verduyn et al., 2007). The ionic strength of the solutions was adjusted to $180 \mathrm{mM}$ with K-propionate. The $\mathrm{pCa}$, i.e., $-\log _{10}\left[\mathrm{Ca}^{2+}\right]$, of the relaxing and activating solution ( $\mathrm{pH} 7.1$ ) was 9 and 4.5, respectively. Solutions with intermediate free $\left[\mathrm{Ca}^{2+}\right]$ were obtained by mixing of the activating and relaxing solutions. Isometric force was measured after the preparation was transferred from relaxing to activating solution, by moving the stage of the inverted microscope. When steady force was reached, the myocyte was reduced in length by $20 \%$ within $2 \mathrm{~ms}$ using the piezoelectric motor and restretched after $30 \mathrm{~ms}$ (slack test). As a result of this intervention, force first dropped to zero and then quickly redeveloped to the original steady-state level. Subsequently, the myocyte was returned to the relaxing solution, and a second slack test (10 s duration) was performed allowing 
the determination of passive force $\left(F_{\text {pas }}\right)$. Active isometric force was calculated from the force level in activating solution $-F_{\text {pas }}$. After maximal activation (pCa 4.5) 4-5 measurements were carried out at submaximal $\left[\mathrm{Ca}^{2+}\right]$ followed by a maximal activation. Force values obtained in solutions with submaximal $\left[\mathrm{Ca}^{2+}\right]$ were normalized to the interpolated maximal force values.

\section{TITIN ISOFORM COMPOSITION}

To determine titin isoform composition, thawed tissue samples were homogenized in a modified Laemmli buffer (Krüger et al., 2010), heated ( $3 \mathrm{~min}$ at $96^{\circ} \mathrm{C}$ and centrifuged. Samples were separated on agarose-strengthened $1.8 \%$ sodium dodecyl sulfate polyacrylamide gels followed by SYPRO Ruby total-protein staining. All samples were run in triplicate. The stiff N2B and compliant N2BA titin isoforms were scanned and quantified by densitometry LAS-4000 system and Multi Gauge V3.2 software (Fuji Science Imaging Systems), mean values for each heart tissue were calculated, and the "mean of the mean" was determined for all groups (EPI and ENDO in sham and MI). Titin isoform composition is presented in relative values $(\mathrm{N} 2 \mathrm{~B}+\mathrm{N} 2 \mathrm{BA}=100 \%)$.

\section{MYOFILAMENT PHOSPHOPROTEOME}

EPI and ENDO tissue ( $\sim 0.5-1.0 \mathrm{mg}$ dry weight) from needle biopsies was tri-chloro acetic acid (TCA) - treated as described previously (Zaremba et al., 2007). Phosphorylation status of myofilament proteins was determined using Pro-Q Diamond phosphostaining (Molecular Probes). In short, samples were separated on a gradient gel (Criterion tris- $\mathrm{HCl} 4-15 \%$ gel, BioRad) and proteins were stained for $1 \mathrm{~h}$ with Pro-Q Diamond Phosphoprotein Stain. Fixation, washing and de-staining were performed according to the manufacturers guidelines. Staining was visualized using the LAS-3000 Image Reader (FUJI; $520 \mathrm{~nm} / 575$ nm Ex/Em; 2 min illumination) and signals were analyzed with AIDA software (Raytest). All protein signals were within the linear range. Subsequently gels were stained overnight with SYPRO Ruby stain (Molecular Probes) and visualized with the LAS-3000 (460 nm/605 nm Ex/Em; 2 s illumination). Since illumination of Pro-Q Diamond-stained gels for $2 \mathrm{~s}$ did not reveal any signal, the signals obtained upon SYPRO Ruby staining are not tainted by Pro-Q signals.

\section{STATISTICAL ANALYSIS}

Data are given as mean \pm SEM. Baseline $(\mathrm{BL})$ data in both groups were compared using two-way (group $\times$ layer) ANOVA, followed by post hoc Bonferroni analysis. Dobutamine effects were tested using three-way (dobu $\times$ group $\times$ layer $)$ followed by two-way (dobu $\times$ layer within each group) ANOVA followed by post hoc paired or unpaired $t$-testing with Bonferroni correction, as appropriate. Significance was accepted when $P<0.05$.

\section{RESULTS}

\section{CARDIAC REMODELING AND PUMP FUNCTION}

Three weeks after myocardial infarction, significant LV remodeling had occurred, reflected in significant dilation, and hypertrophy of the surviving LV myocardium (Table 1). LV systolic dysfunction was evidenced by reductions in ejection fraction and $\mathrm{LVdP} / \mathrm{dt}_{\max }$. Impaired diastolic function was evident from the increased Tau (early diastole) and increased LVEDP (late diastole) in MI compared to sham animals. Pulmonary artery pressure
Table 1 | Anatomical and hemodynamical data measured with closed-chest.

$\operatorname{SHAM}(n=12) \quad \mathrm{MI}(n=12)$

\begin{tabular}{lll}
\hline ANATOMICAL DATA & & \\
BW (kg) & $30 \pm 1$ & $30 \pm 1$ \\
LV weight (g) & $88 \pm 5$ & $102 \pm 3^{*}$ \\
LV weight/BW (g/kg) & $2.9 \pm 0.1$ & $3.5 \pm 0.1^{*}$ \\
LV end-diastolic area $\left(\mathrm{mm}^{2}\right.$ ) & $913 \pm 54$ & $1342 \pm 133^{*}$ \\
LV end-systolic area (mm²) & $426 \pm 37$ & $830 \pm 114^{*}$ \\
2D-Ejection fraction (\%) & $53 \pm 3$ & $39 \pm 4^{*}$ \\
HEMODYNAMIC DATA & & \\
Cardiac output (L/min) & $3.7 \pm 0.3$ & $3.0 \pm 0.1^{*}$ \\
Heart rate (HR, bpm) & $112 \pm 5$ & $124 \pm 12$ \\
Mean aortic pressure (mmHg) & $92 \pm 5$ & $98 \pm 4$ \\
Mean pulmonary artery pressure (mmHg) & $18 \pm 1$ & $28 \pm 2^{*}$ \\
LVdP/dtmax (mmHg/s) & $2002 \pm 119$ & $1717 \pm 68^{*}$ \\
LVdP/dt min (mmHg/s) & $-2199 \pm 164$ & $-1915 \pm 104$ \\
Tau (ms) & $34 \pm 1$ & $45 \pm 4^{*}$ \\
LV end-diastolic pressure (mmHg) & $6 \pm 2$ & $15 \pm 2^{*}$
\end{tabular}

$n$, Number of animals; $B W$, body weight; $L V$, left ventricle. ${ }^{*} P<0.05 ; M I$ vs. sham in one-tailed Student's t-test.

was significantly increased in the MI group, likely as a result of pulmonary congestion (Table 1).

\section{NO TRANSMURAL DIFFERENCES IN FORCE CHARACTERISTICS IN EITHER SHAM OR MI HEARTS}

Force measurements were performed at a sarcomere length of $2.2 \mu \mathrm{m}$ in single Triton-permeabilized cardiomyocytes from six sham hearts (14 EPI and 14 ENDO cells; 2-3 cells/cardiac sample) and from six MI hearts (19 EPI and 21 ENDO cells; 25 cells/cardiac sample). Cardiomyocyte data were averaged per heart and the group averages, shown in Figure 1, were based on the mean values per heart. No differences were found between maximal force $\left(F_{\max }\right.$; Figure 1A), passive force $\left(F_{\text {pas }}\right.$; Figure 1B), $\mathrm{Ca}^{2+}$-sensitivity of force ( $\mathrm{pCa}_{50}$; Figure 1C), and steepness of the force-calcium relation $(\mathrm{nH})$ between EPI and ENDO cells from either sham (respectively, $3.41 \pm 0.14$ and $3.25 \pm 0.17$ ) or (respectively, $3.12 \pm 0.19$ and $2.75 \pm 0.25$ ) hearts.

\section{DIFFERENCES IN FORCE CHARACTERISTICS BETWEEN SHAM AND MI Maximal force-generating capacity}

Force measurements at maximal calcium activation revealed a significantly lower maximal force development in ENDO cells from MI compared to sham hearts (Figure 1A). $F_{\text {max }}$ tended to be somewhat lower in EPI cells from MI hearts as well, but this difference was not statistically significant $(P>0.20)$.

\section{Passive stiffness and titin isoform composition}

Increased passive stiffness of cardiomyocytes has been associated with high LVEDP and diastolic dysfunction of the heart (Borbély et. al, 2005). Although differences in $F_{\text {pas }}$ between EPI and ENDO cells within each group were not significant, a diverse transmural pattern in cardiomyocyte stiffness was observed in sham and MI hearts evident from a trend toward higher $F_{\text {pas }}$ in ENDO 
compared to EPI in sham, while the opposite was observed in MI (Figure 1B; interaction $P<0.05$ in two-way ANOVA). Compared to EPI cells from sham, EPI cells from MI hearts showed a significantly higher $F_{\text {pas }}$.

To investigate if changes in titin isoform composition underlie the observed differences in passive force, titin analyses were performed using agarose-strengthened SDS-PAGE as shown in Figure 2A. Titin is composed of two isoforms, the stiff N2B, and the more compliant N2BA isoform. Our analyses revealed a significantly higher proportion of the stiff $\mathrm{N} 2 \mathrm{~B}$ isoform in EPI compared to ENDO, both in sham and MI $(P<0.05$, EPI vs. ENDO in two-way ANOVA). There was a trend toward increased N2B-proportion in MI EPI vs. Sham EPI $(P=0.17)$, but not in MI ENDO vs. Sham ENDO (Figure 2B).

\section{Myofilament $\mathrm{Ca}^{2+}$-sensitivity}

Force measurements were performed at maximal and submaximal calcium concentrations to determine $\mathrm{Ca}^{2+}$-sensitivity of myofilaments. A significantly higher $\mathrm{pCa}_{50}$ was found in both layers of $\mathrm{MI}$ hearts compared to sham (Figure 1C). No significant differences were found in the steepness of the force-calcium relation between sham and MI cardiomyocytes.
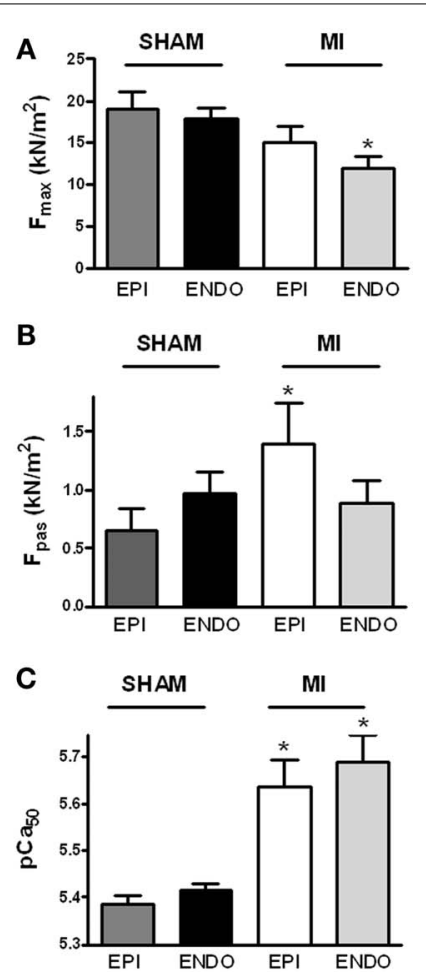

FIGURE 1 | Sarcomeric function. Force measurements were performed at a sarcomere length of $2.2 \mu \mathrm{m}$ in Triton-permeabilized EPI and ENDO cells from six sham and six $\mathrm{Ml}$ hearts. Force characteristics did not differ between EPI and ENDO cells within each group. (A) Maximal force $\left(F_{\max }\right)$ was significantly lower in ENDO cells from MI compared to sham, while passive force [(B) $\left.F_{\text {pas }}\right]$ was significantly higher in EPI cells from $\mathrm{MI}$ compared to sham. (C) In both EPI and ENDO cells, $\mathrm{Ca}^{2+}$-sensitivity $\left(\mathrm{pCa}_{50}\right)$ was significantly higher in $\mathrm{MI}$ than in sham. ${ }^{*} P<0.05, \mathrm{MI}$ vs. sham.

\section{TRANSMURAL PROTEIN COMPOSITION}

Figure 3 shows EPI and ENDO samples separated by 1D-gel electrophoresis from post-infarct remodeled myocardium taken at $\mathrm{BL}$ and during $\beta$-adrenergic receptor stimulation with low (Dob2) and high (Dob10) concentrations of dobutamine. Gels were stained with SYPRO Ruby to determine total-protein levels (Figure 3A) and with Pro-Q Diamond stain to assess phosphorylated proteins (Figure 3B). Phosphorylation signals of proteins were normalized to SYPRO-stained cardiac myosin binding protein $\mathrm{C}$ ( $\mathrm{cMyBP}-\mathrm{C})$ to correct for small differences in sample loading on the gel as described before (Zaremba et al., 2007; Duncker et al., 2009). Analysis of sarcomeric protein phosphorylation profiles did not reveal any differences between EPI and ENDO samples in both sham and MI hearts (Figures 4 and 5).

\section{Baseline}

Baseline phosphorylation of the downstream targets of the $\beta$ adrenergic receptor, cMyBP-C and cardiac troponin (cTnI), did not differ between sham and MI samples (Figure 4). In addition,

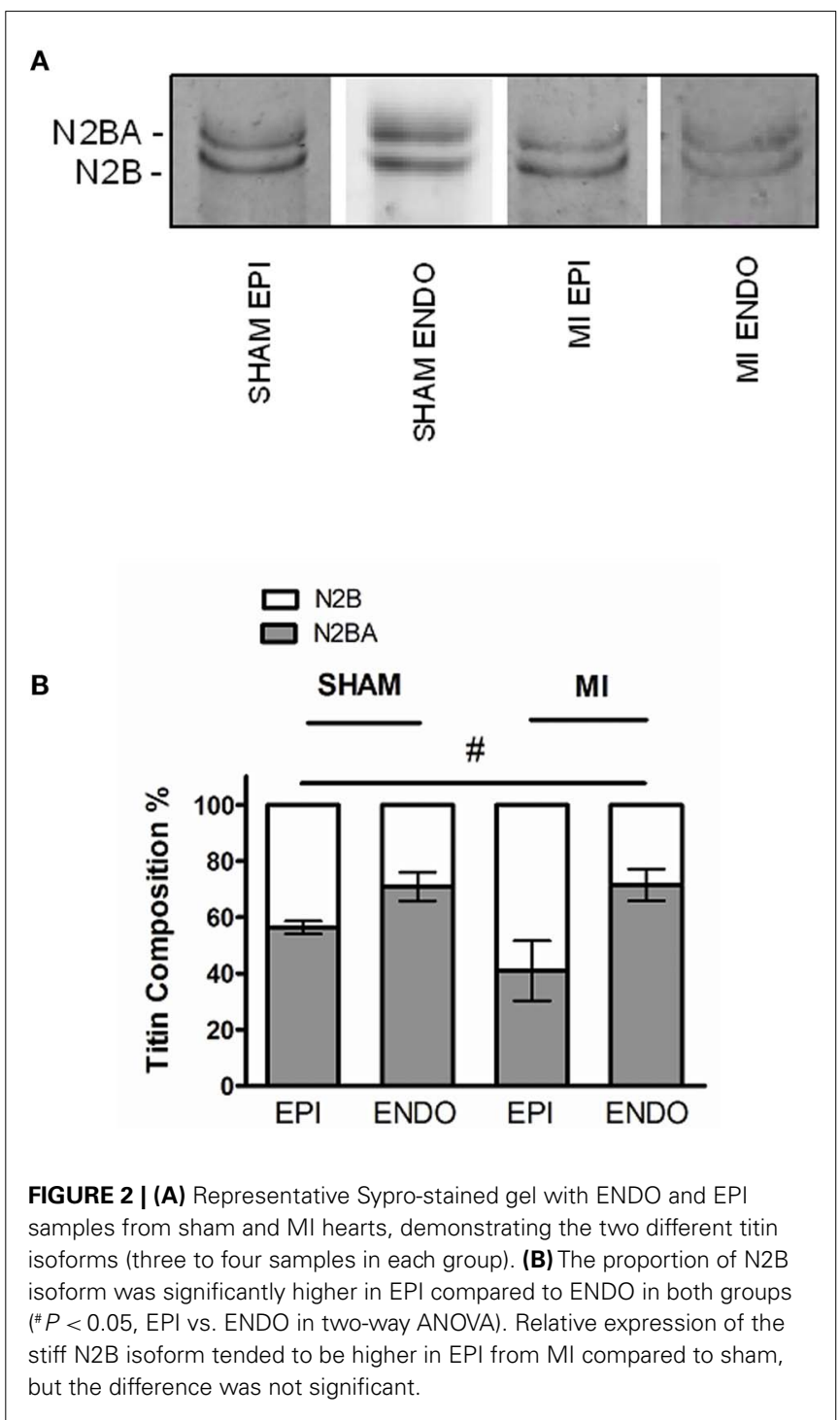




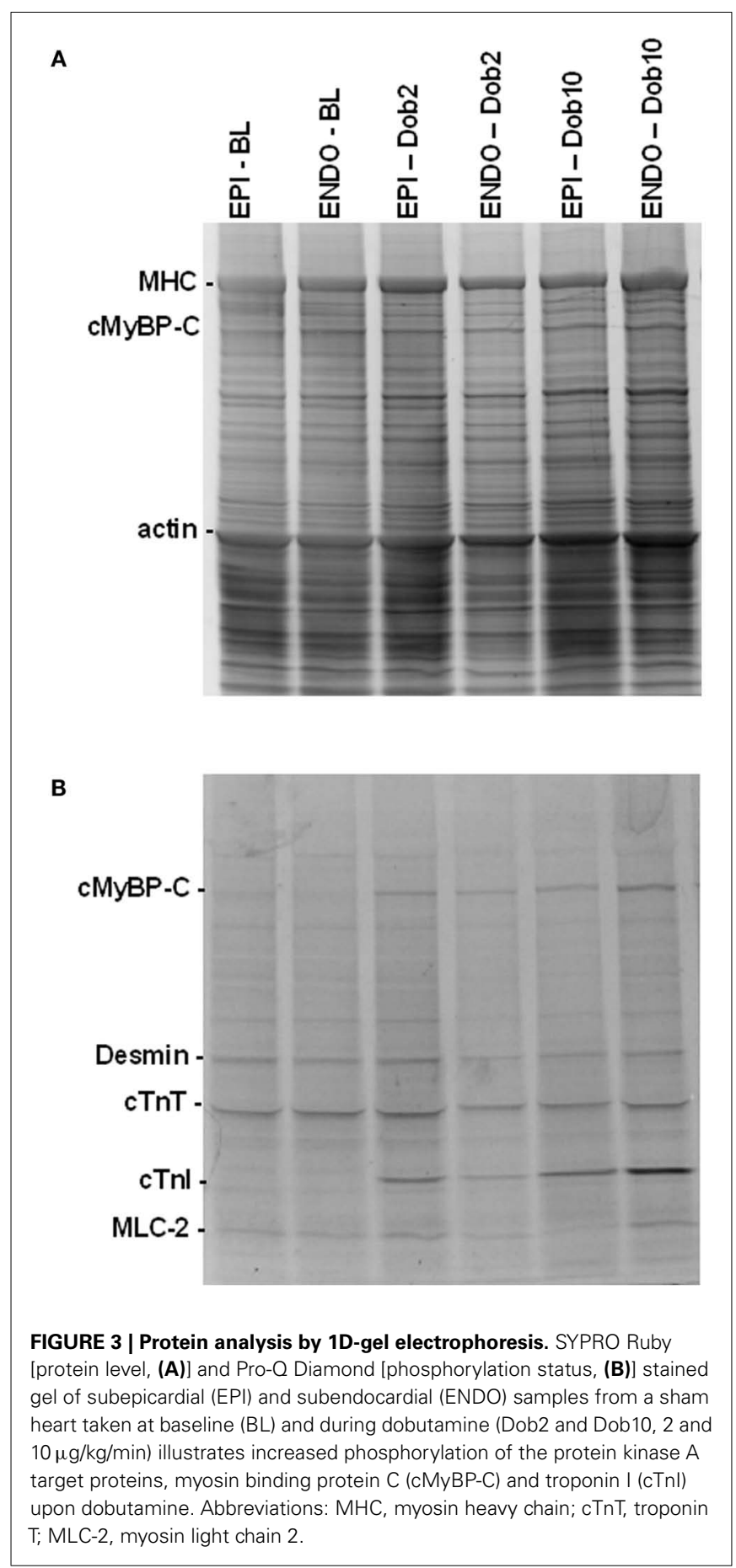

no differences in troponin $\mathrm{T}$ and desmin phosphorylation were found between sham and MI hearts (not shown). However, MLC2 phosphorylation in MI myocardium was significantly lower in both EPI and ENDO samples compared to sham (Figure 4).

\section{Effect of dobutamine}

Dobutamine increased phosphorylation of cMyBP-C and cTnI evident from the Pro-Q Diamond-stained gel in Figure 3. The increase in cMyBP-C phosphorylation was similar in EPI and
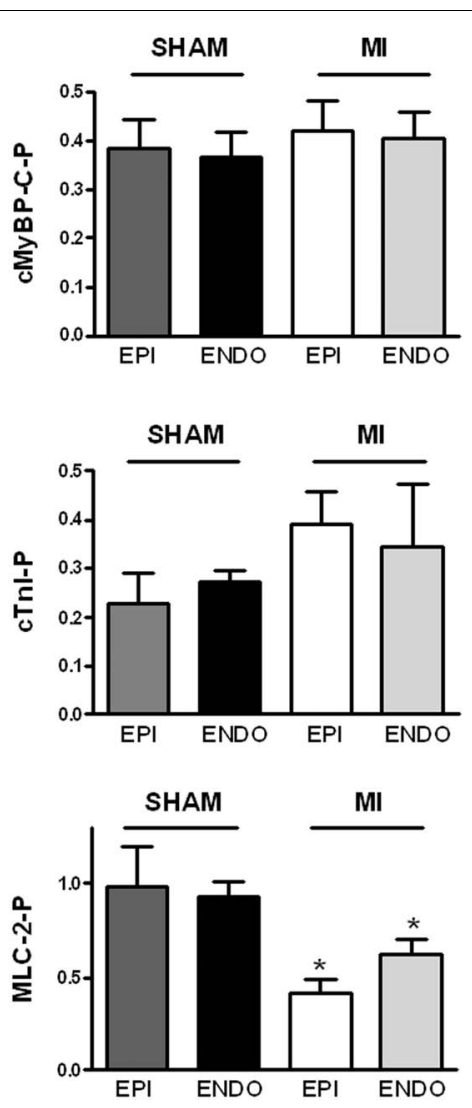

FIGURE 4 | Baseline protein phosphorylation. No differences were found in phosphorylation level of cardiac myosin binding protein (cMyBP-C), troponin I (cTnl), and myosin light chain 2 (Mlc-2) between subepicardial (EPI) and subendocardial (ENDO) samples from 6 Sham en $6 \mathrm{MI}$ swine. MLC-2 phosphorylation was significantly lower in both EPI and ENDO of MI hearts compared to sham. $n$, number of heart samples. ${ }^{*} P<0.05, \mathrm{MI}$ vs. sham.

ENDO samples from both MI and sham hearts (Figure 5). However, although dobutamine significantly increased cTnI phosphorylation in both EPI and ENDO of remodeled post-MI myocardium, the magnitude of the increase was lower compared to sham: a fourfold increase in MI compared to a ninefold increase in sham (Figure 5). Phosphorylation of MLC-2 slightly, but significantly increased in sham myocardium, while no effect was observed in MI (Figure 5). As a consequence, after $\beta$-adrenergic receptor stimulation with dobutamine MLC-2 phosphorylation remained significantly lower in MI compared to sham.

\section{DISCUSSION}

Our study revealed transmural changes in sarcomeric properties 3 weeks after myocardial infarction in a large animal model. Although the functional sarcomeric properties were not different between subepi- and subendo-cardial layers of healthy sham hearts, myocardial infarction induced diverse changes in myocardial function in the EPI and ENDO layers of the remodeled ventricles. A significant reduction in maximal force was observed solely in ENDO, while passive cardiomyocyte stiffness was significantly 

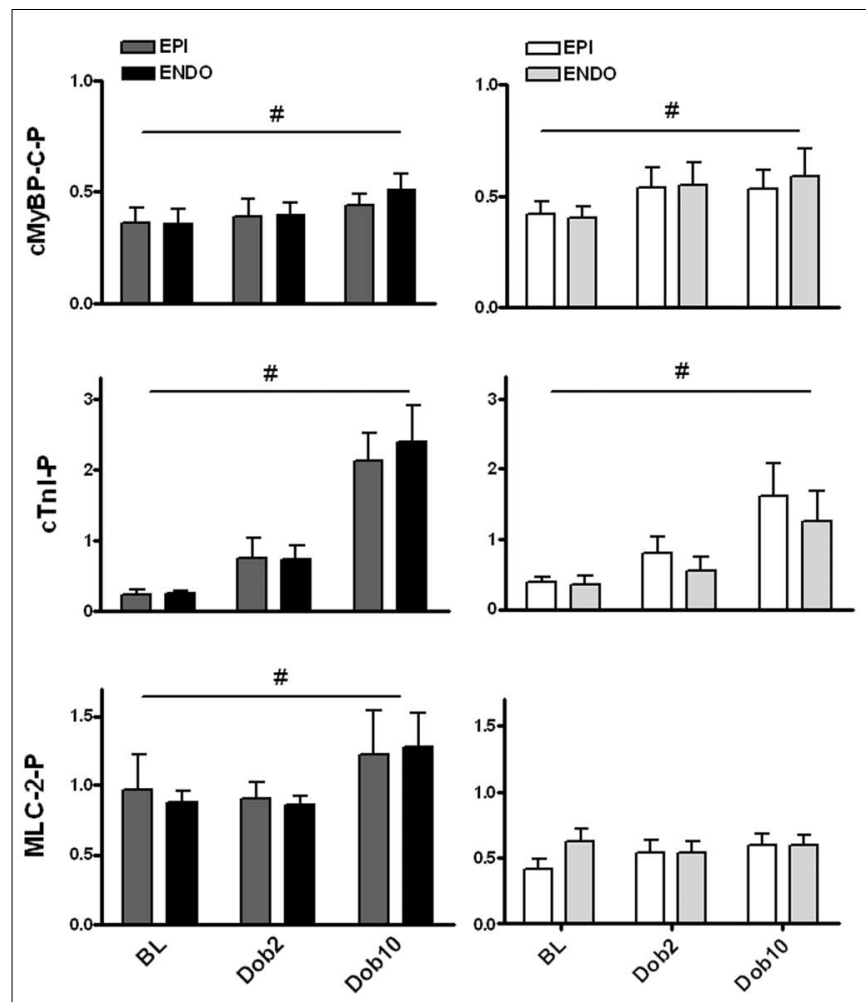

FIGURE 5 | Dobutamine-induced changes in protein phosphorylation. Transmural samples were taken at baseline and after dobutamine stimulation from the same heart (six hearts in each group). Transmural biopsies were cut to obtain subepicardial (EPI) and subendocardial (ENDO) tissue samples. Dobutamine significantly increased phosphorylation of cardiac myosin binding protein C (CMyBP-C) to a similar extent in sham and $\mathrm{MI}$ myocardium. The dobutamine-induced increase in troponin I (cTnl) phosphorylation was larger in sham compared to $\mathrm{MI}$ myocardium for both EPI and ENDO tissue. A significant increase in myosin light chain 2 (MLC-2) phosphorylation was observed in sham myocardium upon dobutamine, which was not observed in MI samples. For all proteins no differences were observed between EPI and ENDO samples of the heart. ${ }^{\#} P<0.05$, effect dobutamine in two-way ANOVA.

increased only in EPI. The implications of these findings will be discussed.

\section{TRANSMURAL SARCOMERIC PROPERTIES IN THE HEALTHY PIG HEART}

No significant transmural differences were observed in functional properties and protein phosphorylation of the sarcomeres across the LV wall in healthy pig hearts. In line with our data, Stelzer et al. (2008) did not find differences in sarcomeric protein phosphorylation and BL functional properties such as maximal and passive force and $\mathrm{Ca}^{2+}$-sensitivity of force development between ENDO and EPI samples from pigs. In rat studies, a higher passive force development was observed in ENDO compared to EPI cells (Cazorla et al., 2000a, 2005; Ait Mou et al., 2008). In our study, passive force tended to be higher in ENDO compared to EPI cells in the sham group, but the difference was not significant. Conversely, a transmural difference in passive force in rat myocardium was evident at a relatively high sarcomere length of $2.3 \mu \mathrm{m}$, but was not seen at $2.1 \mu \mathrm{m}$ (Cazorla et al., 2005). Our functional data were obtained at a sarcomere length of $2.2 \mu \mathrm{m}$, which may explain the absence of a significant difference in passive force between EPI and ENDO layers in healthy myocardium. Unexpectedly, then, our titin analyses showed a lower proportion of the stiff N2B titin isoform in ENDO compared to EPI (Figure 2B). Similarly, Cazorla et al. (2000b) reported significantly higher levels of the N2B isoform in EPI compared to ENDO layers in pig myocardium. As passive stiffness of the sarcomeres is based on isoform composition and phosphorylation status of titin (Krüger and Linke, 2009; LeWinter and Granzier, 2010), a higher passive stiffness in ENDO compared to EPI layers of the heart may involve differences in titin phosphorylation.

In addition to the transmural differences in functional sarcomeric properties in rodent studies, a transmural gradient in MLC-2 phosphorylation was found both in mice and rat (Davis et al., 2001; Aiit Mou et al., 2009). The difference between large mammals and rodents may be explained by the differences in BL phosphorylation among species, as BL phosphorylation values are relatively low in pigs compared to rat and mice (Hamdani et al., 2008). In addition, a recent study by Scruggs et al. (2010) revealed two phosphorylation sites on MLC-2 in mouse myocardium (Serines 14 and 15), while only one phosphorylation site (Serine 15) was found in human cardiac tissue. Increases in MLC-2 phosphorylation have been found upon increased stretch (Cazorla et al., 2005), increased heart rate (Silver et al., 1986; Fitzsimons et al., 1989; Lamberts et al., 2007), and/or upon $\beta$-adrenergic activation of the heart (Westwood and Perry, 1981; Scruggs et al., 2009). In large mammals, transmural phosphorylation differences may only become apparent upon cardiac activation at higher protein phosphorylation levels. To address this possibility, we analyzed the transmural phosphorylation pattern in our pig model upon stimulation of the $\beta$-adrenergic receptor pathway with dobutamine. Apart from increased phosphorylation of the protein kinase A (PKA) target proteins cMyBP-C and cTnI, dobutamine stimulation also increased MLC-2 phosphorylation in healthy sham pigs, consistent with previous studies in rodents (Westwood and Perry, 1981; Scruggs et al., 2009). However, also during strong $\beta$-adrenergic receptor stimulation, no differences in protein phosphorylation were observed between EPI and ENDO samples indicating that a transmural gradient from the subepicardial to subendocardial layer in MLC-2 phosphorylation, but also in cMyBP-C, cTnI, is not a major determinant of myocardial pump function in large mammals.

\section{TRANSMURAL DIFFERENCES IN SARCOMERIC PROPERTIES AFTER MYOCARDIAL INFARCTION}

Myocardial infarction induced diverse changes in maximal and passive force development of cells isolated from remodeled EPI and ENDO layers, while in both layers a significant increase in myofilament $\mathrm{Ca}^{2+}$-sensitivity was found.

Consistent with our previous studies (Van der Velden et al., 2004; Duncker et al., 2009) force measurements in single cardiac cells revealed depressed maximal force-generating capacity in post-infarct remodeled hearts (Figure 1A). The present study showed a significant reduction in maximal force-generating capacity of cells only in the ENDO layer of post-MI remodeled hearts. The mechanism of reduction in maximal force in heart failure models is a matter of ongoing research, but most likely involves altered phosphorylation of sarcomeric proteins. Belin et al. (2007) 
provided evidence for enhanced protein kinase C-mediated protein phosphorylation in reducing maximal force. In addition, reduced MLC-2 phosphorylation has been associated with a significant reduction in the maximal force of contraction (Scruggs et al., 2009). Reduced MLC-2 phosphorylation was present in both layers of the post-infarct remodeled pig heart. Also in human studies we have observed lower MLC-2 phosphorylation in endstage failing myocardium compared to non-failing donor hearts (Van der Velden et al., 2003). Low MLC-2 phosphorylation has been associated with high phosphatase expression/activities (Neumann et al., 1997; Duncker et al., 2009) and has been linked with depressed contractility and cardiac hypertrophy (Sanbe et al., 1999; Dias et al., 2006). As phosphorylation of MLC-2 exerts an important modulating effect on contractile performance (Colson et al., 2010; Scruggs and Solaro, 2011) both low BL MLC-2 phosphorylation and the absence of an increase in MLC-2 phosphorylation upon $\beta$-adrenergic phosphorylation in post-infarct remodeled myocardium may underlie depressed systolic function (i.e., lower $\mathrm{LVdP} / \mathrm{dt}_{\max }$ and stroke volume) in MI pigs.

We observed a significant increase in passive force development only in EPI cells of post-MI remodeled myocardium, which may involve a change in titin isoform composition or alterations in titin phosphorylation (Borbély et al., 2009; Krüger and Linke, 2009; Williams et al., 2009; LeWinter and Granzier, 2010). Interestingly, Jaber et al. (2008) observed a reduction in the compliant titin isoform N2BA and an increase in myofiber passive stiffness only in the subepicardial layer of dogs with tachypacing-induced heart failure. In line with a significantly higher passive force in EPI cells from MI hearts (Figure 1B), the percentage of stiff N2B in the epicardial layer tended to be higher in MI compared to sham (Figure 2B). As indicated above, altered passive stiffness during cardiac disease may also involve changes in phosphorylation of titin, an issue that warrants further research. Increased passive stiffness of the sarcomeres has been associated with increased LVEDPs and diastolic heart failure (Borbély et al., 2005). The high passive stiffness in EPI cells may in part underlie the significantly increased LVEDP observed in MI pigs (Table 1) and contribute to dysfunction during late diastole.

The enhanced $\mathrm{Ca}^{2+}$-sensitivity, which was observed in both layers, may contribute to early diastolic dysfunction evident from the significant increase in Tau in MI compared to sham animals. In addition to a high $\mathrm{Ca}^{2+}$-sensitivity, perturbations in $\mathrm{Ca}^{2+}$-handling impair myocardial relaxation, which may result from alterations in the $\beta$-adrenergic receptor pathway (recently reviewed by Wittköpper et al., 2011). The increase in myofilament $\mathrm{Ca}^{2+}$-sensitivity after $\mathrm{MI}$ has been ascribed to down-regulation and desensitization of the $\beta$-adrenergic receptor pathway as the high $\mathrm{Ca}^{2+}$-sensitivity in post-infarct cardiomyocytes could be normalized to sham values upon treatment with exogenous PKA (Van der Velden et al., 2004; De Waard et al., 2007). However, consistent with our previous study in subendocardial tissue, in the present study we did not find a significantly lower BL phosphorylation of

\section{REFERENCES}

Ait Mou, Y., le Guennec, J. Y., Mosca,

E., de Tombe, P. P., and Cazorla,

O. (2008). Differential contribution

of cardiac sarcomeric proteins in the myofibrillar force response to stretch. Pflugers. Arch. 457, 25-36.

Ait Mou, Y., Reboul, C., Andre, L., Lacampagne, A., and Cazorla,

the PKA target proteins $\mathrm{cMyBP}-\mathrm{C}$ and $\mathrm{cTnI}$ in subepicardial tissue as well (Figure 4). Phosphorylation of cTnI even tended to be somewhat higher in MI compared to sham samples, which most likely reflects activities of other (possibly up-regulated) kinases. The only evidence for reduced $\beta$-adrenergic receptor signaling was the blunted increase in cTnI phosphorylation in both EPI and ENDO layers of MI hearts compared to sham, indicative for reduced phosphorylation of cTnI at PKA sites (Figure 4). In our previous study, we have shown with Western blot analysis that phosphorylation of PKA sites on $\mathrm{CTnI}$ is indeed lower in MI compared to sham ENDO samples taken during dobutamine stimulation (Boontje et al., 2011). In contrast to cTnI, the increase in cMyBP-C phosphorylation upon dobutamine was similar in MI and sham groups (Figure 5) and may involve activation of $\mathrm{Ca}^{2+}$. dependent calmodulin kinase II to compensate for reduced PKAmediated phosphorylation (Boontje et al., 2011). The exact mechanism underlying enhanced myofilament $\mathrm{Ca}^{2+}$-sensitivity observed in different animal models remains incompletely understood but likely involves site-specific phosphorylation of sarcomeric proteins and has been extensively discussed elsewhere (Marston and de Tombe, 2008; Solaro and van der Velden, 2010).

\section{CONCLUSION}

Our study showed no transmural differences in sarcomere function at BL in sham hearts. In contrast to rodent studies we did not observe a transmural difference in MLC-2 phosphorylation in pig hearts both at $\mathrm{BL}$ and upon dobutamine infusion indicating that a transmural MLC-2 phosphorylation gradient is not prerequisite for proper cardiac pump function in large mammals. We did find a small and significant increase in MLC-2 phosphorylation upon activation of $\beta$-adrenergic receptors in sham hearts, which was absent in MI and may underlie impaired cardiac performance during increased stress in post-infarct hearts.

After MI a significantly lower $F_{\max }$ was observed only in ENDO cells and a higher $F_{\text {pas }}$ in EPI, while myofilament $\mathrm{Ca}^{2+}$ sensitivity was increased in both layers. These heterogeneous transmural changes in sarcomeric properties in post-infarct remodeled myocardium likely contribute differently to systolic vs. diastolic dysfunction. Thus, the reduction in maximal force-generating capacity in ENDO cells may contribute to systolic dysfunction of post-MI remodeled hearts, while the increase in myofilament $\mathrm{Ca}^{2+}$-sensitivity observed in both layers may impair relaxation during the early phase of diastole. Moreover, increased passive stiffness of EPI cells may hinder LV filling in late diastole and in part underlie high LVEDP in post-MI hearts. Together these transmurally heterogenous alterations in sarcomeric properties in post-MI remodeled myocardium may disrupt coordinated contraction and relaxation patterns across the LV wall.

\section{ACKNOWLEDGMENTS}

This study was supported by the Netherlands Heart Foundation (grant 2005B220 to Jolanda van der Velden).

O. (2009). Late exercise training improves non-uniformity of transmural myocardial function in rats with ischaemic heart failure. Cardiovasc. Res. 81, 555-564.
Belin, R. J., Sumandea, M. P., Allen, E. J., Schoenfelt, K., Wang, H., Solaro, R. J., and de Tombe, P. P. (2007). Augmented protein kinase C-alpha-induced myofilament 
protein phosphorylation contributes to myofilament dysfunction in experimental congestive heart failure. Circ. Res. 101, 195-204.

Boontje, N. M., Merkus, D., Zaremba, R., Versteilen, A., de Waard, M. C., Mearini, G., de Beer, V. J., Carrier, L., Walker, L. A., Niessen, H. W., Dobrev, D., Stienen, G. J. M., Duncker, D. J., and van der Velden, J. (2011). Enhanced myofilament responsiveness upon $\beta$-adrenergic stimulation in post-infarct remodeled myocardium. J. Mol. Cell. Cardiol. 50, 487-499.

Borbély, A., Falcao-Pires, I., van Heerebeek, L., Hamdani, N., Edes, I., Gavina, C., Leite-Moreira, A. F., Bronzwaer, J. G. F., Papp, Z., van der Velden, J., Stienen, G. J. M., and Paulus, W. J. (2009). Hypophosphorylation of the stiff $\mathrm{N} 2 \mathrm{~B}$ titin isoform raises cardiomyocyte resting tension in failing human myocardium. Circ. Res. 104, 780-786.

Borbély, A., van der Velden, J., Bronzwaer, J. G. F., Papp, Z., Édes, I., Stienen, G. J. M., and Paulus, W. J. (2005). Cardiomyocyte stiffness in diastolic heart failure. Circulation 111, 774-781.

Cazorla, O., Le Guennec, J. Y., and White, E. (2000a). Length-tension relationships of sub-epicardial and sub-endocardial single ventricular myocytes from rat and ferret hearts. J. Mol. Cell. Cardiol. 32, 735-744.

Cazorla, O., Freiburg, A., Helmes, M., Centner, T., McNabb, M., Wu, Y., Trombitás, K., Labeit, S., and Granzier, H. (2000b). Differential expression of cardiac titin isoforms and modulation of cellular stiffness. Circ. Res. 86, 59-67.

Cazorla, O., Szilagyi, S., Le Guennec, J. Y., Vassort, G., and Lacampagne, A. (2005). Transmural stretchdependent regulation of contractile properties in rat hearts and its alteration after myocardial infarction. FASEB J. 19, 88-90.

Colson, B. A., Locher, M. R., Bekyarova, T., Patel, J. R., Fitzsimons, D. P., Irving, T. C., and Moss, R. L. (2010). Differential roles of regulatory light chain and myosin binding protein-C phosphorylations in the modulation of cardiac force development. J. Physiol. 588, 981-993.

Davis, J. S., Hassanzadeh, S., Winitsky, S., Lin, H., Satorius, C., Vemuri, R., Aletras, A. H., Wen, H., and Epstein, N. D. (2001). The overall pattern of cardiac contraction depends on a spatial gradient of myosin regulatory light chain phosphorylation. Cell 107, 631-641.
De Waard, M. C., van der Velden, J., Bito, V., Ozdemir, S., Biesmans, L., Boontje, N. M., Dekkers, D. H. W., Schoonderwoerd, K., Schuurbiers, J., de Crom, M., Stienen, G. J. M., Sipido, K. R., Lamers, J. M. J., and Duncker, D. J. (2007). Early exercise training normalizes myofilament function and attenuates left ventricular pump dysfunction in mice with a large myocardial infarction. Circ. Res. 100, 1079-1088.

Dias, F. A., Walker, L. A., Arteaga, G. M., Walker, J. S., Vijayan, K., Peña, J. R., Ke, Y., Fogaca, R. T., Sanbe, A., Robbins, J., and Wolska, B. M. (2006). The effect of myosin regulatory light chain phosphorylation on the frequency-dependent regulation of cardiac function. J. Mol. Cell. Cardiol. 41, 330-339.

Duncker, D. J., Boontje, N. M., Merkus, D., Versteilen, A., Krysiak, J., Mearini, G., El-Armouche, A., de Beer, V. J., Lamers, J. M. J., Carrier, L., Walker, L. A., Linke, W. A., Stienen, G. J. M., and van der Velden, J. (2009). Prevention of myofilament dysfunction by beta-blocker therapy in postinfarct remodeling. Circ. Heart Fail. 2, 233-242.

Fitzsimons, D. P., Bodell, P. W., and Baldwin, K. M. (1989). Phosphorylation of rodent cardiac myosin light chain 2: effects of exercise. J. Appl. Physiol. 67, 2447-2453.

Glukhov, A. V., Fedorov, V. V., Lou, Q., Ravikumar, V. K., Kalish, P. W., Schuessler, R. B., Moazami, N., and Efimov, I. R. (2010). Transmural dispersion of repolarization in failing and nonfailing human ventricle. Circ. Res. 106, 981-991.

Haitsma, D. B., Bac, D., Raja, N., Boomsma, F., Verdouw, P. D., and Duncker, D. J. (2001). Minimal impairment of myocardial blood flow responses to exercise in the remodeled left ventricle early after myocardial infarction, despite significant hemodynamic and neurohumoral alterations. Cardiovasc. Res. 52, 417-428.

Hamdani, N., de Waard, M. C., Messer, A. E., Boontje, N. M., Kooij, V., van Dijk, S. J., Versteilen, A., Lamberts, R., Merkus, D., dos Remedios, C., Duncker, D. J., Borbely, A., Papp, Z., Paulus, W. J., Stienen, G. J. M., Marston, S. B., and van der Velden, J. (2008). Myofilament dysfunction in cardiac disease from mice to men. $J$. Muscle Res. Cell. Motil. 29, 189-201.

Jaber, W. A., Maniu, C., Krysiak, J., Shapiro, B. P., Meyer, D. M., Linke, W. A., and Redfield, M. M. (2008). Titin isoforms, extracellular matrix, and global chamber remodeling in experimental dilated cardiomyopathy: functional implications and mechanistic insight. Circ. Heart Fail. 1, 192-199.

Krüger, M., Babicz, K., von FrielingSalewsky, M., and Linke, W. A. (2010). Insulin signaling regulates cardiac titin properties in heart development and diabetic cardiomyopathy. J. Mol. Cell. Cardiol. 48, 910-916.

Krüger, M., and Linke, W. A. (2009). Titin-based mechanical signalling in normal and failing myocardium. $J$. Mol. Cell. Cardiol. 46, 490-498.

Lamberts, R. R., Hamdani, N., Soekhoe, T. W., Boontje, N. M., Zaremba, R., van der Velden, J., and Stienen, G. J. M. (2007). Frequency-dependent $\mathrm{Ca}^{2+}$-desensitization in failing rat hearts. J. Physiol. (Lond.) 582, 695-709.

LeWinter, M. M., and Granzier, H. (2010). Cardiac titin: a multifunctional giant. Circulation 121, 2137-2145.

Litovsky, S. H., and Antzelevitch, C. (1989). Rate dependence of action potential duration and refractoriness in canine ventricular endocardium differs from that of epicardium: the role of the transient outward current. J. Am. Coll. Cardiol. 14, 1053-1066.

Marston, S. B., and de Tombe, P. P. (2008). Troponin phosphorylation and myofilament $\mathrm{Ca}^{2+}$-sensitivity in heart failure: increased or decreased? J. Mol. Cell. Cardiol. 45, 603-607.

Neumann, J., Eschenhagen, T., Jones, L. R., Linck, B., Schmitz, W., Scholz, H., and Zimmermann, N. (1997). Increased expression of cardiac phosphatases in patients with end-stage heart failure. J. Mol. Cell. Cardiol. 29, 265-272.

Rajashree, R., Blunt, B. C., and Hof mann, P. A. (2005). Modulation of myosin phosphatase targeting subunit and protein phosphatase 1 in the heart. Am. J. Physiol. 289, H1736-H1743.

Sanbe, A., Fewell, J. G., Gulick, J., Osinska, H., Lorenz, J., Hall, D. G., Murray, L. A., Kimball, T. R., Witt, S. A., and Robbins, J. (1999). Abnormal cardiac structure and function in mice expressing nonphosphorylatable cardiac regulatory myosin light chain 2. J. Biol. Chem. 274, 21085-21094.

Scruggs, S. B., Hinken, A. C., Thawornkaiwong, A., Robbins, J., Walker, L. A., de Tombe, P. P., Geenen, D. L., Buttrick, P. M., and Solaro, R. J. (2009). Ablation of ventricular myosin regulatory light chain phosphorylation in mice causes cardiac dysfunction in situ and affects neighboring myofilament protein phosphorylation. J. Biol. Chem. 284, 5097-5106.

Scruggs, S. B., Reisdorph, R., Armstrong, M. L., Warren, C. M., Reisdorph, N., Solaro, R. J., and Buttrick, P. M. (2010). A novel, in-solution separation of endogenous cardiac sarcomeric proteins and identification of distinct charged variants of regulatory light chain. Mol. Cell. Proteomics 9, 1804-1818.

Scruggs, S. B., and Solaro, R. J. (2011). The significance of regulatory light chain phosphorylation in cardiac physiology. Arch. Biochem. Biophys. 510, 129-134.

Silver, P. J., Buja, L. M., and Stull, J. T. (1986). Frequency-dependent myosin light chain phosphorylation in isolated myocardium. J. Mol. Cell. Cardiol. 18, 31-37.

Solaro, R. J., and van der Velden, J. (2010). Why does troponin have so many phosphorylation sites? Fact and fancy. J. Mol. Cell. Cardiol. 48, 810-816.

Stelzer, J. E., Norman, H. S., Chen, P. P., Patel, J. R., and Moss, R. L. (2008). Transmural variation in myosin heavy chain isoform expression modulates the timing of myocardial force generation in porcine left ventricle. J. Physiol. (London) 586, 5203-5214.

Van der Velden, J., Merkus, D., Klarenbeek, B. R., James, A. T., Boontje, N. M., Dekkers, D. H., Stienen, G. J. M., Lamers, J. M., and Duncker, D. J. (2004). Alterations in myofilament function contribute to left ventricular dysfunction in pigs early after myocardial infarction. Circ. Res. 95, e85-e95.

Van der Velden, J., Papp, Z., Zaremba, R., Boontje, N. M., de Jong, J. W., Owen, V. J., Burton, P. B. J., Goldmann, P., Jaquet, K., and Stienen, G. J. M. (2003). Increased $\mathrm{Ca}^{2+}$. sensitivity of the contractile apparatus in end-stage human heart failure results from altered phosphorylation of contractile proteins. Cardiovasc. Res. 57, 37-47.

Van Kats, J. P., Duncker, D. J., Haitsma, D. B., Schuijt, M. P., Niebuur, R., Stubenitsky, R., Boomsma, F., Schalekamp, M. A., Verdouw, P. D., and Danser, A. H. (2000). Angiotensin-converting enzyme inhibition and angiotensin II type 1 receptor blockade prevent cardiac remodeling in pigs after myocardial infarction: role of tissue angiotensin II. Circulation 102, 1556-1563.

Verduyn, S. C., Zaremba, R., van der Velden, J., and Stienen, G. J. M. 
(2007). Effects of contractile protein phosphorylation on force development in permeabilized rat cardiac myocytes. Basic Res. Cardiol. 102, 476-487.

Westwood, S. A., and Perry, S. V. (1981). The effect of adrenaline on the phosphorylation of the $\mathrm{P}$ light chain of myosin and troponin $\mathrm{I}$ in the perfused rabbit heart. Biochem. J. 197, 185-193.

Williams, L., Howell, N., Pagano, D., Andreka, P., Vertesaljai, M., Pecor, T., Frenneaux, M., and Granzier, H. (2009). Titin isoform expression in aortic stenosis. Clin. Sci. 117, 237-242.

Wittköpper, K., Dobrev, D., Eschenhagen, T., and El-Armouche, A. (2011). Phosphatase-1 inhibitor-1 in physiological and pathological $\beta$ adrenoceptor signalling. Cardiovasc. Res. 91, 392-401.

Zaremba, R., Merkus, D., Lamers, J. M. J., Paulus, W. J., dos Remedios, C., Duncker, D. J., Stienen, G. J. M., and van der Velden, J. (2007). Quantitative analysis of myofilament protein phosphorylation in small cardiac biopsies. Proteomics Clin. Appl. 1, 1285-1290.
Conflict of Interest Statement: The authors declare that the research was conducted in the absence of any commercial or financial relationships that could be construed as a potential conflict of interest.

Received: 07 July 2011; accepted: 28 October 2011; published online: 24 November 2011.

Citation: van der Velden, Merkus D, de Beer V, Hamdani $N$, Linke WA, Boontje NM, Stienen GJM and Duncker DJ (2011) Transmural heterogeneity of myofilament function and sarcomeric protein phosphorylation in remodeled myocardium of pigs with a recent myocardial infarction. Front. Physio. 2:83. doi: 10.3389/fphys.2011.00083

This article was submitted to Frontiers in Striated Muscle Physiology, a specialty of Frontiers in Physiology.

Copyright () 2011 van der Velden, Merkus, de Beer, Hamdani, Linke, Boontje, Stienen and Duncker. This is an open-access article subject to a nonexclusive license between the authors and Frontiers Media SA, which permits use, distribution and reproduction in other forums, provided the original authors and source are credited and other Frontiers conditions are complied with. 